Document downloaded from:

http://hdl.handle.net/10251/83359

This paper must be cited as:

Clement, J.; Torregrosa, G.; Hervás-Peralta, J.; Barrera Vilar, D.; Sales Maicas, S.; Fernandez-Pousa, CR. (2016). Interrogation of a Sensor Array of Identical Weak FBGs using Dispersive Incoherent OFDR. IEEE Photonics Technology Letters. 28(10):1154-1156. doi:10.1109/LPT.2016.2533163,.

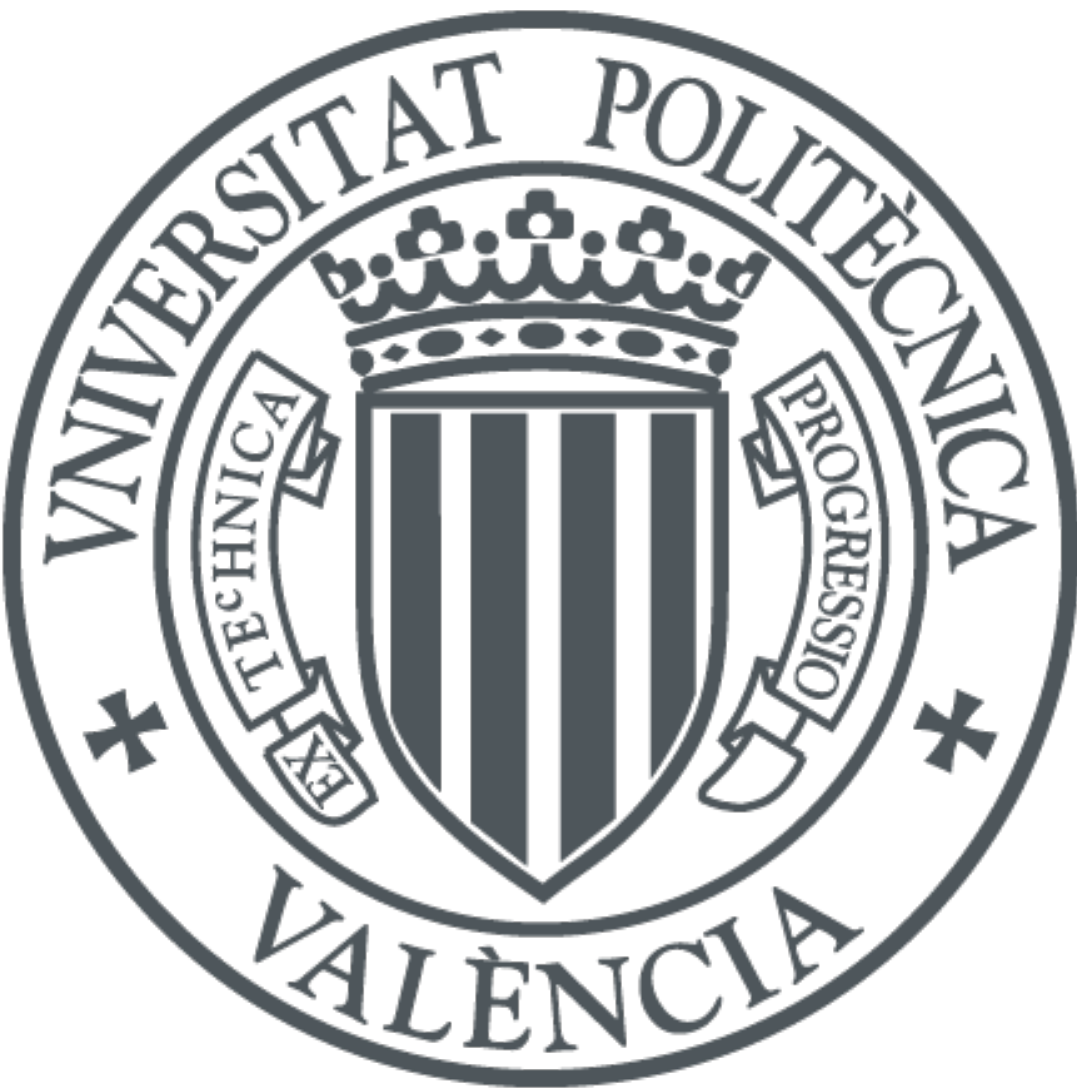

The final publication is available at

http://dx.doi.org/10.1109/LPT.2016.2533163

Copyright Institute of Electrical and Electronics Engineers (IEEE)

Additional Information

(c) 2016 IEEE. Personal use of this material is permitted. Permission from IEEE must be obtained for all other users, including reprinting/ republishing this material for advertising or promotional purposes, creating new collective works for resale or redistribution to servers or lists, or reuse of any copyrighted components of this work in other works. 


\title{
Interrogation of a Sensor Array of Identical Weak FBGs using Dispersive Incoherent OFDR
}

\author{
Juan Clement, Germán Torregrosa, Javier Hervás, David Barrera, Salvador Sales, Senior Member, \\ IEEE, and Carlos R. Fernández-Pousa, Senior Member, IEEE
}

\begin{abstract}
Incoherent Optical Fourier-Domain Reflectometry incorporating a dispersive delay line is used for the interrogation of an array of three identical fiber Bragg gratings with a Bragg wavelength of $1552.81 \mathrm{~nm}$, reflectivity of $-19.3 \mathrm{~dB}$ and $10-\mathrm{cm}$ separation. The dispersive delay line induces different delays in the wavelengths reflected by each grating, thus being sensitive to Bragg wavelength shifts. Compared with conventional incoherent Optical Fourier-Domain Reflectometry, dispersive effects decrease the spatial resolution, which in our experiments reached a value of $1.2 \mathrm{~cm}$ in fiber at a measurement bandwidth of 10 GHz. As a quasi-distributed temperature sensor, the array shows an accuracy of $\pm 0.5^{\circ} \mathrm{C}$ for temperatures up to $100^{\circ} \mathrm{C}$, and an estimated total measurement range of $540^{\circ} \mathrm{C}$. Tradeoffs between bandwidth, scan time, dispersion-dependent spatial resolution, and accuracy, are also analyzed.
\end{abstract}

Index Terms - Optical fiber sensors, OFDR, dispersive delay line, fiber Bragg gratings, sensor interrogation.

$\mathrm{Q}$ UASI-DISTRIBUTED sensing based on arrays of lowreflectivity, identical fiber Bragg gratings (FBGs) offers specific advantages in addition to the general benefits inherent to fiber optics. Identical FBGs can be efficiently fabricated at high spatial density [1], and their low reflectivity allows for crosstalk-free operation even in large arrays of FBGs [2]. In turn, specific interrogation methods are required to demodulate this type of arrays. Spectral analysis cannot detect the wavelength shifts experienced by each of the FBGs in the array and, in order to provide both range and spectral selectivity, either time-multiplexed techniques [1], [3] or optical Fourier-domain reflectometry (OFDR) systems [2], [4] have been developed for this purpose.

More recently, an interrogation concept based on radiofrequency (RF) measurements has been reported [5]. Range selectivity is provided by Fourier transforming the electrical RF response of the array, as in incoherent OFDR (I-OFDR) [6], [7], and spectral dependence is introduced by use of a dispersive delay line (DDL) that maps wavelengths to delays. This concept, which has recently been used in several FBG sensor interrogation systems at microwave frequencies [8][11], benefits from the performance of I-OFDR in terms of spatial resolution, robustness and sensitivity due to the incoherent and electrical narrowband detection of opticallycarried microwave tones, as opposed to the wideband detection necessary for pulsed sources [6], [7]. The frequency span in [5], however, was limited to a bandwidth of $1.1 \mathrm{GHz}$, which lead to a spatial resolution of $10 \mathrm{~cm}$ much greater than the dimension of the FBGs. This fact precludes the use of lowbandwidth schemes in high-density arrays. In addition, [5] is based on the spectral correlation between the FBG reflectivity and a programmable spectral filter, which results in an increase of loss and complexity, and also showed limitations of measurement linearity and range.

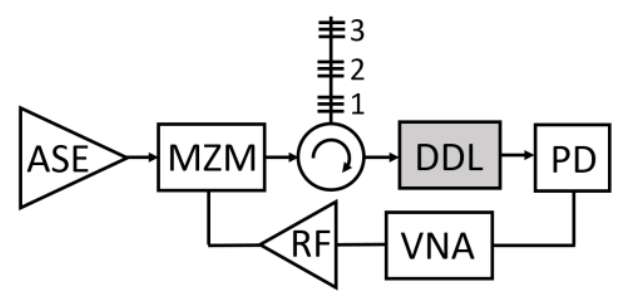

Fig. 1. Scheme of conventional I-OFDR (without Dispersive Delay Line, DDL) and the proposed technique (with DDL). MZM: Mach-Zehnder modulator, PD: photodiode, RF: wideband amplifier.

In this Letter, we report on the use of a simpler scheme where these limitations are overcome. As in [5], the method is a modification of step-frequency I-OFDR by the incorporation of a DDL, as is schematically depicted in Fig. 1. The reflectors are illuminated by an amplitude-modulated wave and, after retrieving the system's RF transfer function $H_{R F}(f)$ by use of a Vector Network Analyzer (VNA), the impulse response is numerically computed by inverse Fourier transformation. In the impulse response of I-OFDR, all the wavelengths reflected by a given event contribute to a peak in a definite temporal position whose location is simply the time delay undergone by the modulating RF tone, or the group delay experienced by the optical wave. After the insertion of the DDL, group delays become a function of wavelength and the reflected spectrum is resolved in time. Using the same principle, changes in Bragg wavelengths can be detected as differential delays: if the $k$-th grating, initially at Bragg wavelength $\lambda_{k}$ and with delay $\tau\left(\lambda_{k}\right)$, experiences a shift up to $\lambda^{\prime}{ }_{k}$, the resulting delay is:

$$
\tau\left(\lambda_{k}^{\prime}\right)=\tau\left(\lambda_{k}\right)+D_{k} \times\left(\lambda_{k}^{\prime}-\lambda_{k}\right),
$$

where $D_{k}=d \tau_{k} / d \lambda$ is the total dispersion, measured in ps/nm, in the $k$-th optical circuit. In our setup, the modulated wave is directly detected after dispersion and processed as in I-OFDR, not being used the spectral correlation stage employed in [5].

In a proof-of-principle experiment, we applied this method to the interrogation of a quasidistributed temperature sensor array composed of three equal FBGs mutually separated by 10 $\mathrm{cm}$. The gratings, of $2.5 \mathrm{~mm}$ in length, were centered at 
$1552.81 \mathrm{~nm}$, had a FWHM $\Delta \lambda=0.41 \mathrm{~nm}$, and an average peak reflectivity of $-19.3 \mathrm{~dB}$, as is inferred from the reflectivity plot in the inset of Fig. 2. The reflectivity sideband at $\lambda>1554$ $\mathrm{nm}$ is due to a fabrication error in the last grating, as will be elucidated below by use of the present technique.

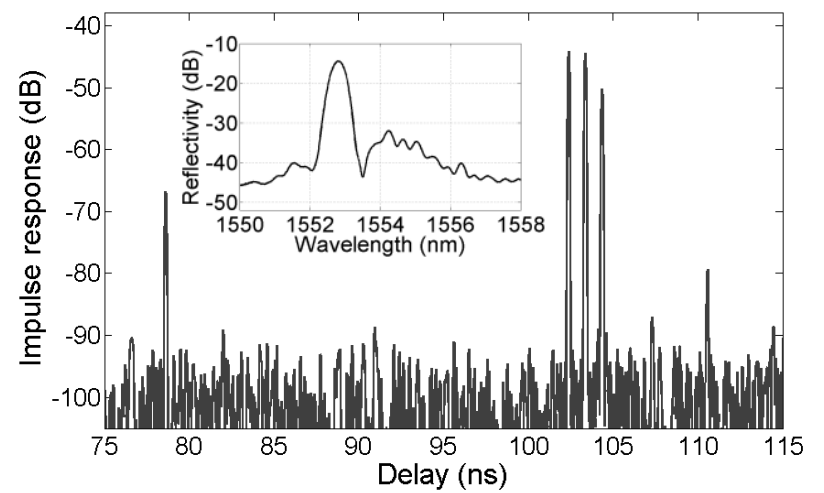

Fig. 2. I-OFDR trace of the sensor array $(B W=10 \mathrm{GHz}, I F B W=10 \mathrm{~Hz}$, 2001 points per trace, scan time $377 \mathrm{~s}$ ). The peaks correspond, from left to right, to the input connector, to the sensor array, and to the fiber end. The physical distance from the input connector to the first FBG is $240 \mathrm{~cm}$, and to the fiber end, $325 \mathrm{~cm}$. The separation between gratings is $10 \mathrm{~cm}$. Inset: reflectivity spectrum of the array.

We first probed the FBG array with a conventional I-OFDR setup, using a 20-nm ASE source, a 20-GHz push-pull MZM and a 50-GHz photodiode. The RF level at the modulator input was boosted up to $+11 \mathrm{dBm}$ to increase the modulation index by use of a wideband 14-GHz RF amplifier, and the RF transfer function was measured in a bandwidth $(B W)$ of 10 $\mathrm{GHz}$ by use of a VNA (Keysight Fieldfox N9928). The impulse response was determined by its time-domain software after windowing the double-sided RF response with a KaiserBessel window with $\beta=6.5$. The results are shown in Fig. 2, where we used the standard back-to-back reference trace [7], [11]. The impulse response is retrieved in a temporal range given by the inverse of the frequency scanning step of the VNA trace, $T_{R}=(\Delta f)^{-1}$, which was set to $200 \mathrm{~ns}$ to visualize all the reflective features in the array. When $\beta=6.5$, the FWHM of the peaks in the impulse response is $\Delta \tau_{I-O F D R}=$ $1 / B W$, in agreement with the measured value of $100 \mathrm{ps}$. This leads to a spatial resolution $\Delta x$ of $1 \mathrm{~cm}$ in fiber, sufficient to resolve the FBGs in the array. The additional peaks in Fig. 2 are due to the input connector and to the fiber end, which was intentionally broken in order to minimize end reflections.

Next, we inserted a DDL following the scheme in Fig. 1. We used a dispersion-compensating, low-ripple chirped FBG (CFBG, Proximion) in the reflection band 1540-1560 nm. It has an insertion loss of $1.8 \mathrm{~dB}$ at $1552.81 \mathrm{~nm}$, and a total dispersion $D=-170 \mathrm{ps} / \mathrm{nm}$. Since fiber dispersion between consecutive FBGs can be neglected, this is the value of dispersion experienced by the reflected waves in all the FBGS in the array. In order to obtain an alias-free impulse response, and thus an ordered sequence of reflectors as in Fig. 2, and also to minimize the acquisition time, we used a reference trace that sets the temporal origin closer to the beginning of the array. The contribution to the response of the most distant reflector at delay $\tau$ is of the form $H_{R F}(f) \sim \exp (-j 2 \pi f \tau)$, and absence of temporal alias requires that the frequency step verifies $\Delta f<(2 \tau)^{-1}$. A reference reflector at $\tau_{0}$ changes the transfer function to $\exp \left[-j 2 \pi f\left(\tau-\tau_{0}\right)\right]$ and the requirement is relaxed to $\Delta f<\left[2\left(\tau-\tau_{0}\right)\right]^{-1}$, allowing for wider frequency steps and faster acquisition times. We took the reference trace without the CFBG and by replacing the sensor array by a $1-\mathrm{m}$ patchcord with a PC-polished end, thus setting the temporal zero at the delay corresponding to its end point. In our experiments, $T_{R}=40 \mathrm{~ns}$, sufficient to account for the added distance in the FBG array and the CFBG.

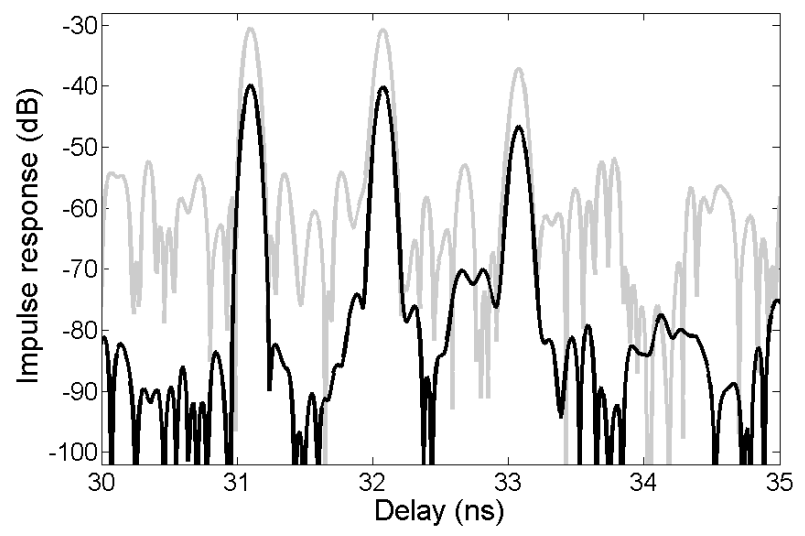

Fig. 3. Impulse responses of the sensor array (single sweep, $B W=10 \mathrm{GHz}$, 401 points per trace). Black trace, IF $B W=10 \mathrm{~Hz}$, scan time $83 \mathrm{~s}$. Gray trace: IF $B W=1 \mathrm{kHz}$, scan time $1.4 \mathrm{~s}$. The gray trace is shifted $10 \mathrm{~dB}$ upwards.

In Fig. 3, with a black trace, we show the three FBG peaks in the impulse response after the insertion of the array and the CFBG, retrieved in a single sweep with $B W=10 \mathrm{GHz}$. In the third grating we observe a sidelobe at delays $\sim 32.75 \mathrm{~ns}$. This temporal sidelobe is consequence of the temporal spread induced by dispersion of the reflection band at $\lambda>1554 \mathrm{~nm}$ shown in the inset of Fig. 2. Dispersive effects also widen the peaks in the impulse response. Their FWHM $\Delta \tau$ can be estimated as:

$$
\Delta \tau^{2}=\Delta \tau_{I-O F D R}^{2}+(D \Delta \lambda)^{2},
$$

where the second term describes the FWHM of the temporal spread induced by the dispersion of the reflected wavelengths. The measured $\Delta \tau$ is $120 \mathrm{ps}$, in accord with the value given by (2). The resulting spatial resolution in fiber is $\Delta x=1.2 \mathrm{~cm}$.

Subsequently, we explored the possibility of tracking Bragg wavelength shifts through the peak of the impulse response. The third, most distant FBG was heated, free of strain, in a 5$\mathrm{cm}$ oven, and the temperature monitored by use of a thermocouple placed in contact with the grating. The delay was measured at temperatures up to about $100{ }^{\circ} \mathrm{C}$. The small delay shifts were determined with the VNA configured with a display resolution of $0.5 \mathrm{ps}$. The results are plotted in the top row of Fig. 4, showing a high linearity with slope $-1.63 \mathrm{ps} /{ }^{\circ} \mathrm{C}$ or, equivalently, a sensitivity of the Bragg wavelength shift of $9.6 \mathrm{pm} /{ }^{\circ} \mathrm{C}$. The same experiment was repeated in the first grating with similar results. The sensor accuracy, given by the standard deviation $\sigma$ of the residuals in the linear fit, reaches the accuracy of our thermocouple, $\pm 0.5^{\circ} \mathrm{C}$. This amounts to a 
delay deviation of $\pm 0.8 \mathrm{ps}$. The $2 \sigma$ value is of the order of the maximum peak-to-peak group delay ripple ( $\sim 3.5 \mathrm{ps})$ shown by the CFBG in the band of interest, a value that can be considered as a worst-case estimate of the temporal accuracy in the wavelength-to-time mapping provided by CFBG.

The measurement range can be estimated from the worst scenario where a FBG is heated whereas the adjacent is not, so that the peaks are resolved if they are at least separated by $\Delta \tau$ [6]. With our delay separation between FBGs $\Delta t \cong 1 \mathrm{~ns}$, the allowed excursion in Bragg wavelengths, $\delta \lambda$, is given by $\Delta t$ $\dashv D \mid \delta \lambda>\Delta \tau$. This yields $\delta \lambda<5.2 \mathrm{~nm}$ or, equivalently, a temperature range $\delta T<540{ }^{\circ} \mathrm{C}$.

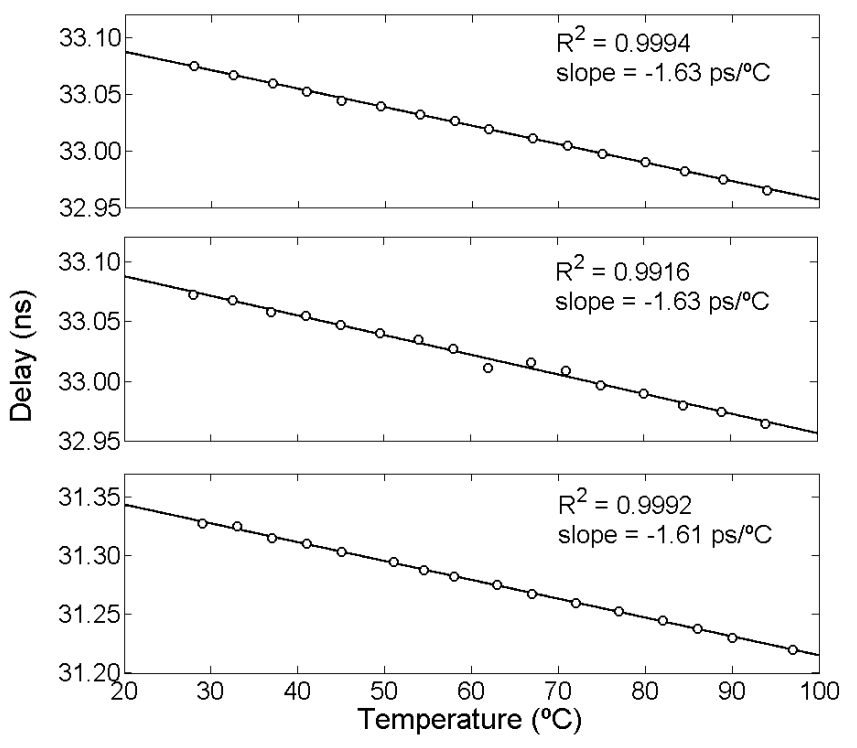

Fig. 4. Delay variation with temperature. From top to bottom: third grating with $B W=10 \mathrm{GHz}$ and $I F B W=10 \mathrm{~Hz}$, third grating with $B W=10 \mathrm{GHz}$ and IF $B W=1 \mathrm{kHz}$, and first grating with $B W=2.5 \mathrm{GHz}$ and $I F B W=10 \mathrm{~Hz}$.

Scan time can be reduced by increasing the intermediate frequency bandwidth (IF $B W$ ) at the expense of accuracy. To illustrate this tradeoff, we repeated the experiment increasing the $I F B W$ from $10 \mathrm{~Hz}$ to $1 \mathrm{kHz}$, thus increasing the RF noise level by $20 \mathrm{~dB}$, as is shown in Fig 3 . The scan time is reduced to $1.4 \mathrm{~s}$, and the variation of the third peak with temperature, plotted in the central row of Fig. 4, shows a decrease in accuracy to $\pm 2{ }^{\circ} \mathrm{C}$. This result also represents the expected performance when $I F B W$ is kept at $10 \mathrm{~Hz}$ and the decrease in SNR is due to FBG peak reflectivities of $-30 \mathrm{~dB}$.

Scan time can also be reduced by decreasing the acquisition $B W$ at the expense of spatial resolution. The impulse response for $B W=2.5 \mathrm{GHz}$ is plotted in Fig. 5, displaying the expected reduction of spatial resolution $(\Delta \tau=410 \mathrm{ps})$. We performed a final test by heating the first grating. The results, in the bottom row of Fig. 4, indicate that the accuracy is maintained $\left( \pm 0.6^{\circ} \mathrm{C}\right)$.

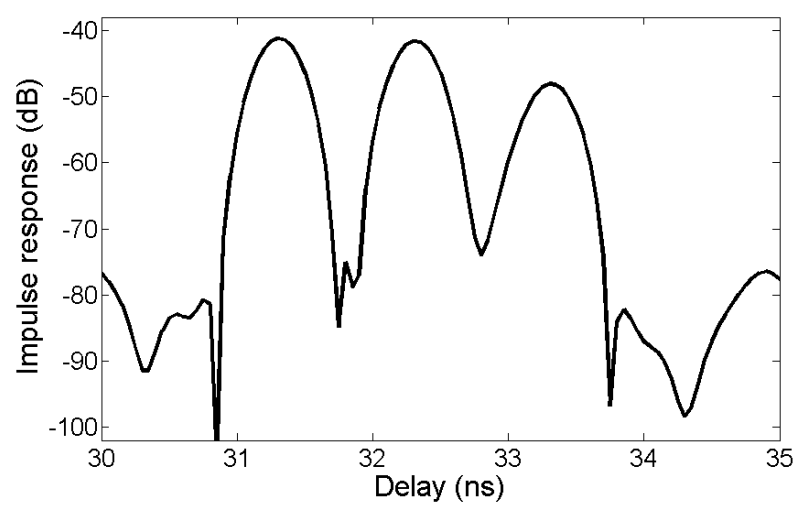

Fig. 5. Impulse response of the sensor array: single sweep, $B W=2.5 \mathrm{GHz}$, IF $B W=10 \mathrm{~Hz}, 101$ point per trace, scan time $22 \mathrm{~s}$.

In summary, we have interrogated an array of identical, weak FBGs as a temperature sensor by use of a dispersive IOFDR technique, where changes in Bragg wavelengths are mapped into delays through the insertion of a dispersive delay line. The FBG reflectivity of about $-20 \mathrm{~dB}$ and the obtained spatial resolution of $1.2 \mathrm{~cm}$ surpass by almost an order of magnitude the values of previously reported related methods. Weaker reflectivities could be probed by the additional use of trace averaging, optical amplification, or of sources with higher power.

\section{REFERENCES}

[1] Y. Wang et al., "A large serial time-division multiplexed fiber Bragg grating sensor network," J. Lightwave Technol., vol. 30, no. 17, pp. 2751 $-2756,2012$.

[2] B. A. Childers et al., "Use of 3000 Bragg grating strain sensor distributed on four eight-meter optical fibers during static load tests of a composite structure," Proc. SPIE, vol. 4332, pp. 133-142, 2001.

[3] M. Froggatt and J. Moore, "Distributed measurement of static strain in an optical fiber with multiple Bragg gratings at nominally equal wavelengths," Appl. Opt., vol. 37, no. 10, pp. 1741-1746, 1998.

[4] C. Hu et al., "A novel interrogation system for large scale sensing network with identical ultra-weak fiber Bragg gratings," J. Lightwave Technol., vol. 32, no. 7, pp. 1406-1411, 2014.

[5] L. Xia et al., "Identical FBG-based quasi-distributed sensing by monitoring the microwave responses," IEEE Photon, Technol. Lett., vol. 27, no. 3, pp. 323-325, 2015.

[6] D. Derickson, Fiber optic test and measurement. Upper Saddle River, N.J.: Prentice Hall PTR, 1998.

[7] D. W. Dolfi et al., "5-mm-resolution optical-frequency-domain reflectometry using a coded phase-reversal modulator," Opt. Lett., vol. 13, no. 8, pp. 678-680, 1988.

[8] A. L. Ricchiuti et al., "Long fiber Bragg grating sensor interrogation using discrete-time microwave photonic filtering techniques," Opt. Express, vol. 21, no. 23, pp. 28175-28181, 2013.

[9] R. Cheng et al., "Radio frequency FBG-based interferometer for remote adaptive strain monitoring," IEEE Photon, Technol. Lett., vol. 27, no. 15, pp. 1577-1580, 2015.

[10] J. Hervás et al., "An interrogation technique of FBG cascade sensors using wavelength to radio-frequency delay mapping," J. Lightwave Technol., vol. 33, no. 11, pp. 2222-2227, 2015.

[11] J. Clement and C. R. Fernández-Pousa, "Spectral analysis using a dispersive microwave photonics link based on a broadband chirped fiber Bragg grating," J. Lightwave Technol., vol. 33, no. 20, pp. 4207-4214, 2015. 